\title{
Impact of a public sensitization campaign on migraine management in France
}

\author{
Anne Donnet · Michel Lantéri-Minet · Gilles Géraud • \\ Evelyne Guegan-Massardier · Geneviève Demarquay · \\ Pierric Giraud - Christian Lucas · Dominique Valade
}

Received: 19 March 2009/Accepted: 6 May 2009/Published online: 30 May 2009

(c) Springer-Verlag 2009

\begin{abstract}
Migraine is a common and frequently disabling condition. Nevertheless, many migraine sufferers do not consult for migraine, are not medically followed up and self-treat the attacks. "Tour de France of migraine" consisted of free-access conferences held in six large towns in France following a wide public information campaign. This sensitization campaign aimed at providing participants with educational information on migraine disease and on current therapies. Headache sufferers were then invited to respond to two consecutive questionnaires delivered at the end of the conferences and 3 months later to assess the
\end{abstract}

\section{A. Donnet $(\square)$}

Department of Neurology, La Timone Hospital,

264 Boulevard Saint-Pierre, 13385 Marseille Cedex, France

e-mail: anne.donnet@mail.ap-hm.fr; adonnet@ap-hm.fr

\section{Lantéri-Minet}

Department of Evaluation and Treatment of Pain,

Pasteur University Hospital, Nice, France

G. Géraud

Department of Neurology, Rangueil Hospital, Toulouse, France

E. Guegan-Massardier

Department of Neurology, Rouen Hospital, Rouen, France

G. Demarquay

Department of Functional Neurology and Epileptology,

Neurological Hospital, Lyon, France

P. Giraud

Department of Neurology, Annecy Hospital, Annecy, France

C. Lucas

Neurological Clinic, Salengro Hospital, Lille, France

D. Valade

Emergency Headache Centre, Lariboisière Hospital,

Paris, France influence of the information delivered on their migraine management. Tour de France of migraine recruited mainly severe migraine sufferers, most of whom had already consulted and were medically followed up. However, migraine management was often suboptimal in these subjects since most of them found their acute treatment of attacks ineffective and only few of them received a prophylactic treatment. Three months after the conferences, more than half of respondents had consulted for headaches. There was a significant improvement in migraine-related disability, as reflected by a significant decrease in mean Headache Impact Test 6-item score, which might have been related to the higher proportion of subjects receiving a prophylactic treatment of migraine. The Tour de France of migraine campaign revealed the difficulty in sensitizing migraine sufferers towards the necessity of being medically followed up. Mainly patients with severe migraine attended the conferences and derived clinical benefit from the educational program. Other strategies should be developed to reach a wider population of migraine sufferers.

Keywords Migraine - Medical follow-up ·

Burden of migraine $\cdot$ Patient education

\section{Introduction}

Migraine is a frequent condition affecting about $11 \%$ of adult populations worldwide [1], yet many patients remain undiagnosed or untreated. A nationwide population-based epidemiologic survey carried out in France indicated that $60 \%$ of subjects diagnosed as having migraine are not aware that they suffer from this disease [2]. Moreover, although migraine is associated with high levels of disability and impairment of health-related quality of life, 
many migraine sufferers $(50-80 \%)$ are not medically followed up and self-treat their attacks [2-4]. The disability associated with migraine is an important target for treatment, and effective acute and preventative treatments exist, but some of them are available by prescription only. Importantly, the absence of medical control may promote overuse of acute treatments, which may lead to medicationoveruse headache, a condition often refractory to treatment [5].

Recently, the World Health Organization (WHO) in collaboration with three international associations (World Headache Alliance, International Headache Society, European Headache Federation) initiated "Lifting the burden", a global campaign to reduce the burden of headache worldwide [6]. The campaign central tenet is that the healthcare solution for headache in most areas of the world is education. Educational programs directed towards the general public and patients consulting general practitioners, aiming to raise awareness of headache disorders and their consequences are considered essential for the diagnosis and management of migraine and other headache disorders. Studies carried out in the setting of a health maintenance organization or specialized headache clinic indeed showed that addition of therapeutic patient education to routine medical management improves the outcome of migraine or tension-type headaches [7, 8]. Reinforcement of information of the general population may also improve migraine diagnosis and management for those subjects who are not aware of their disease or not medically followed up.

In this context, a series of conferences on migraine was organized in several towns of France ("Tour de France of migraine") by the French Society for the Study of Migraine and Headache (SFEMC) to sensitize the general public towards migraine. These conferences targeted migraine sufferers as well as their close relations and aimed at providing information about migraine disease and the possibilities of current proper management of migraine. Headache sufferers were invited to respond to two consecutive questionnaires: the first one to establish their headache characteristics as they attended the conference, the second one 3 months later to assess the influence of the conference on the management of their headaches. Results from this study are presented below.

\section{Patients and methods}

\section{Educational program}

The sensitization campaign on migraine was initiated by the SFEMC in association with the French Association of Private Neurologists (ANLLF). It was conducted from
September to October 2007 in six towns of France: Lyon, Rouen, Lille, Marseille, Toulouse and Paris ("Tour de France of migraine"). The conferences intended for the general public took place on a Saturday morning and were free of registration fees. Each conference consisted of four seminars delivered by migraine specialists and dealing with definition, epidemiology, treatment and management of migraine, with a particular focus on the relationship between migraine and female hormones. The seminars were given with the help of visual aids which were elaborated by the SFEMC and the same for all towns. Seminars were then followed by debates. Information for attending the conferences was circulated via local media, via the SFEMC website and by means of public notices within 2 weeks beforehand.

Study questionnaires

The initial self-administered paper questionnaire was handed out at the end of the conference. It was especially intended for the individuals who said that they suffered from headaches. Three months later, all respondents to the first questionnaire who had provided their particulars and given their consent were contacted by phone by the polling institute TNS Healthcare and asked to respond to a second simplified questionnaire.

The initial 24-item study questionnaire queried about(1) the reason why the subject attended the conference: because he/she or someone amongst his/her close relations was suffering from headaches, or only because he/she was interested in health information; (2) subjects' demographics: age, sex; (3) attributes of headaches: persistent or recurrent quality, number of days per month, existence of aura symptoms before the attacks; (4) migraine diagnostic criteria for migraine without aura according to the International Classification of Headache Disorders, 2nd edition (ICHD-II), category 1.1 [9]; (5) headache-related disability as evaluated by the Headache Impact Test 6-item (HIT-6) assessing the impact of headaches on daily living activities [10]; (6) whether the subject had already consulted specifically for headaches, and if yes, which practitioner; and whether the subject was medically followed up for headaches; (7) the consumption of acute treatments of attacks, the name and dosage of the medication most frequently used as first-line treatment regardless of it was a non-prescription- or a prescription-only medication; (8) whether the subject took a preventive medication for migraine; and (9) whether the subject intended to consult for headaches after having attended the conference.

The medications used for the acute treatment of migraine were classified as recommended (triptans, ergotamine derivatives, non-steroidal anti-inflammatory drugs, aspirin alone or combined with metoclopramide) or non- 
recommended treatments (paracetamol, opioids) according to the French National Authority for Health (HAS) recommendations, while specific treatment included triptans, ergotamine derivatives and aspirin combined with metoclopramide [11]. Effectiveness of the usual treatment of attacks was assessed according to the set of four questions designed by the SFEMC and the HAS. This questionnaire captures information about: (1) significant migraine relief $2 \mathrm{~h}$ after drug intake, (2) treatment tolerability, (3) single drug intake to treat an attack and (4) rapid resumption of normal social, family or professional activities. "Yes" responses to all four questions characterized treatment effectiveness [11, 12].

The second 16-item study questionnaire, administered by telephone, mainly consisted of the sections 5 and 7 of the initial questionnaire. In addition, the second questionnaire queried about whether the subject had consulted for headaches since the conference, and if yes, which practitioner was consulted.

\section{Ethical approval}

Nominative data were recorded and subjects' written informed consent was required to participate in the second phase of the survey. Therefore, study protocol was submitted to and approved by the French Commission on Data Processing and Liberties (CNIL). Participants received no compensation.

Data processing and statistical analysis

Responses to both questionnaires were processed by the polling institute TNS Healthcare Sofres. Categorical variables were summarized by the percentages in the corresponding categories. Numerical variables were summarized by the usual descriptive statistics (mean, SD). Statistical comparisons of data between subsets of subjects (e.g. subjects with or without medical follow-up, initial vs. second questionnaire) were performed using the $Z$ test. Statistical significance was considered at $P<0.05$.

\section{Results}

\section{Survey population}

A total of 143 questionnaires were collected during the sensitization campaign (Phase 1 of the survey) including one blank questionnaire. One hundred and twenty respondents gave their particulars to be contacted by phone 3 months after the conference (Phase 2 of the survey). Of them, 105 could be actually contacted and accepted to respond to the second questionnaire. The remaining 15 subjects gave false particulars or did not respond to phone call $(n=6)$, refused to participate to the second phase of the survey $(n=5)$ or stated that they were not migraine sufferers $(n=4)$.

Phase 1 of the survey: characteristics of the subjects participating in the sensitization campaign

The population of individuals attending the conferences was mainly made up of women $(74 \%)$ and of headache sufferers $(80 \%)$. Most participants $(95 \%)$ mentioned that suffering from headaches was the main reason for attending the conference. Amongst the 114 participants who stated that they suffered headaches, $110(96 \%)$ were true migraine sufferers according to the ICHD-II diagnostic criteria, with a marked preponderance of subjects with strict migraine (77 subjects, 70\%) (code 1.1) over those with probable migraine (code 1.6). Most migraine sufferers were women (79\%) and the majority (64\%) had migraine without aura. On average, the number of days per month with headaches was $7.3 \pm 6.8$ (range $1-31$ ), with $18 \%$ of subjects having chronic daily headaches ( $>15$ days/ month). The majority of migraine sufferers responding to the initial questionnaire (70 subjects; 64\%) were medically followed up. Migraine sufferers with medical follow-up comprised significantly more women (85 vs. 68\%; $P<0.05)$ and fewer subjects aged 55-64 years (10 vs. 34\%; $P<0.01)$. Most migraine sufferers considered their migraine as disabling, and 100/110 (95\%) had a HIT-6 score above 55. Mean HIT-6 score was $66.9 \pm 5.9$ (range 49-76) and significantly higher for the migraine sufferers who were medically follow-up $(67.8 \pm 5.5$ vs. $65.2 \pm 6.4$ for those without medical follow-up; $P<0.05)$. More migraine sufferers with HIT-6 score $>55$ were medically followed up (95 vs. $82 \%$ for those without medical followup; $P<0.05)$. Table 1 summarizes the data on migraine management at the time of the conference for all migraine sufferers participating in survey Phase 1. Nearly, all migraine sufferers, either medically followed up or not, had already consulted specifically for their headaches, mainly general practitioners or neurologists. More migraine sufferers with medical follow-up than without had consulted a neurologist $(P<0.05)$. Most migraine sufferers used to treat their attacks, mainly with a recommended or a specific treatment. Significantly more migraine sufferers with than without medical follow-up used a recommended or a specific treatment (both $P<0.05$ ) (Table 1 ). The mean number of medication units (tablets, capsules, suppositories, etc.) taken to treat an attack was $2.3 \pm 2.0$, with the majority of migraine sufferers taking a single (49\%) or two medication units $(26 \%)$. Only $11 \%$ of them took five or more medication units, mainly those not medically followed up (21 vs. 5\% of those with medical follow-up; 
Table 1 Migraine management for subjects responding to the initial questionnaire (Phase 1)

\begin{tabular}{|c|c|c|c|}
\hline $\begin{array}{l}\text { Characteristics of } \\
\text { migraine sufferers, } n(\%)\end{array}$ & $\begin{array}{l}\text { All subjects, } \\
N=110\end{array}$ & $\begin{array}{l}\text { Medically followed up } \\
N=70\end{array}$ & $\begin{array}{l}\text { Not medically } \\
\text { followed up }{ }^{\mathrm{b}}, N=39\end{array}$ \\
\hline Previous consultation for headaches & $107(97 \%)$ & $70(100 \%)$ & $36(93 \%)^{*}$ \\
\hline General practitioner & $98(91 \%)$ & & \\
\hline Neurologist & $62(58 \%)$ & $43(62 \%)$ & $16(41 \%)^{*}$ \\
\hline Ophthalmologist & $28(26 \%)$ & & \\
\hline Gynaecologist & $26(25 \%)$ & & \\
\hline Otorhinolaryngologist & $7(6 \%)$ & & \\
\hline Others & $19(17 \%)$ & & \\
\hline Acute treatment of attacks & $107(97 \%)$ & & \\
\hline Specific treatment ${ }^{\mathrm{a}}$ & $63(59 \%)$ & $50(72 \%)$ & $12(32 \%)^{*}$ \\
\hline Non-specific treatment ${ }^{\mathrm{a}}$ & $39(36 \%)$ & & \\
\hline Recommended treatment $^{\mathrm{a}}$ & $83(77 \%)$ & $61(87 \%)$ & $22(56 \%)^{*}$ \\
\hline Non-recommended treatment ${ }^{\mathrm{a}}$ & $19(18 \%)$ & & \\
\hline Not specified & $5(5 \%)$ & & \\
\hline Prophylactic treatment of migraine & $27(25 \%)$ & $25(35 \%)$ & $3(7 \%)^{*}$ \\
\hline \multicolumn{4}{|l|}{ Intention to consult } \\
\hline Yes & $23(21 \%)$ & & \\
\hline No & $11(10 \%)$ & & \\
\hline Missing data & $6(5 \%)$ & & \\
\hline Not applicable (already followed up) & $70(64 \%)$ & & \\
\hline
\end{tabular}

$N$ indicates the number of migraine sufferers amongst the participants who responded to the initial questionnaire at the time of the conference (Phase 1)

${ }^{a}$ According to the recommendations of the French National Authority for Health (HAS) for migraine medical management (adapted from Ref. [11])

${ }^{\mathrm{b}}$ Only the characteristics showing statistically significant differences $(* P<0.05$ vs. migraine sufferers medically followed up) between the two subsets of migraine sufferers are shown in these columns

$P<0.05)$. On the whole, the acute treatment of attacks was found effective in only 40 of 93 migraine sufferers with assessable responses at the SFEMC-HAS set of four questions (43\%). Only one-quarter of migraine sufferers was currently taking a preventive medication for migraine, mainly those medically followed up $(P<0.05$ vs. migraine sufferers without medical follow-up). Of the migraine sufferers not already medically followed up, two-thirds intended to consult for migraine soon (Table 1).

Phase 2 of the survey: influence of the sensitization campaign on migraine management

Of the 94 migraine sufferers participating in survey Phase 2, 49 (52\%) consulted within the 3 months following the conferences. Significantly more migraine sufferers previously medically followed up consulted compared with those without medical follow-up (67 vs. $27 \% ; P<0.05$ ). Most consulters consulted general practitioners (32 subjects, 65\%) and/or neurologists (19 subjects, 38\%). Onethird of the non-consulters (34\%) were migraine sufferers who intended to consult at the end of the conferences. All migraine sufferers with pronounced migraine-related disability at the time of the conferences (HIT-6 score $>60$ ) had consulted. In the whole group of migraine sufferers who completed the HIT-6 questionnaire at the end of the conferences and 3 months later, mean HIT-6 score decreased significantly from $66.9 \pm 5.9$ to $63.4 \pm 8.3$ $(P<0.05)$. However, the observed decrease in mean HIT-6 score was of a similar size whether migraine sufferers had consulted or not (Fig. 1). The proportion of migraine sufferers with HIT- 6 score $\geq 60$ decreased from 100 to $86 \%(-14 \%)$ in those who consulted and from 83 to $58 \%(-25 \%)$ in those who did not. Regarding acute treatments of attacks, no significant change was observed between the two phases of the survey. Three months after the conference, most migraine sufferers $(93 \%)$ used medications to treat the attacks, mainly specific and recommended treatments (Fig. 2). Migraine sufferers medically followed up still used more specific and recommended treatments than those without medical follow-up. The mean number of medication units taken to treat an attack $(2.3 \pm 2.0$ initially; $2.2 \pm 2.63$ months later) and the proportion of migraine sufferers with effective treatment 


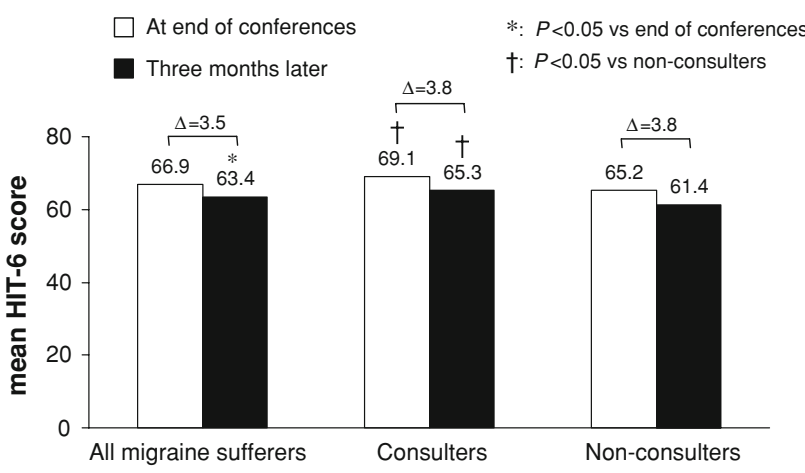

Fig. 1 Headache Impact Test 6-item scores at end of conferences (white bars) and 3 months later (black bars) in the whole group of migraine sufferers and according to medical consultation 3 months later. Open triangle denotes difference between the mean HIT-6 scores recorded at the end of conferences and 3 months later. ${ }^{*} P<0.05$ versus end of conferences, ${ }^{\dagger} P<0.05$ versus nonconsulters

did not differ significantly between the two phases of the survey (Fig. 2). In contrast, 3 months after the conferences, the proportion of migraine sufferers taking a preventive medication for migraine was significantly larger than initially $(P<0.05$; Fig. 2$)$.

\section{Discussion}

Although migraine is a frequent disease associated with considerable disability, it remains largely unrecognized and improperly treated. The objective of Tour de France of migraine was to sensitize migraine sufferers and their close relations to the necessity of seeking the care of a physician to benefit from the current most effective therapies and avoid the risk of medication overuse. Despite a large information campaign, only a low proportion of headache sufferers attended the conferences and it appears that most of those who did generally had a more severe disease were already medically followed up. Furthermore, the influence of the information delivered on migraine sufferers' behaviour and migraine management 3 months after the conferences seems to have been rather limited.

Migraine (strict or probable) affects more than one in five subjects in France (21\%) [13]. The conferences organized in six important towns in France only recruited 142 headache sufferers that accepted to participate in the survey, of whom 110 were recognized as true migraine sufferers. This low rate of participation conforms well to the notion that migraine sufferers show defeatism and resignation towards medical care of their disease and their willingness to manage their headaches by themselves [14]: a preceding survey indicated that $28 \%$ of migraine sufferers in France never consulted for migraine and $41 \%$ lapsed consulting [4]. Insufficient patient information, as reflected by the high proportion of migraine sufferers not self-aware that they have migraine [2], may be the cause of their unwillingness to seek medical care. Other factors may include the fact that migraine attacks are relatively short and rarely observed by the physicians, to whom they are described retrospectively and succinctly or even not reported at all, while the patients continue their own way to manage the attacks with the notion that only themselves have a good knowledge of their attacks. They would eventually consult a physician only to get a prescription, which would entail the risk of altering the normal patientphysician relationship [15].

The population who actually attended such conferences was mainly composed of headache sufferers $(80 \%$ of subjects), most of them ( $96 \%$ of headache sufferers) being true migraine sufferers as defined by the ICHD-II diagnostic criteria for migraine. Moreover, the population of migraine sufferers recruited via this campaign consisted mainly of severe migraine sufferers, as attested by the HIT6 score: most migraine sufferers had a HIT- 6 score $>55$ (range 49-76). The severity of the disease may explain the moderate impact of this campaign.

Similar observations were made in a sensitization campaign (Casilino study) on unrecognized migraine that was conducted in Italy in 2003 [16]. Headache sufferers
Fig. 2 Use of acute and preventive treatments of migraine at end of conferences (white bars) and 3 months later (black bars) in the whole group of migraine sufferers. $* P<0.05$ versus end of conferences

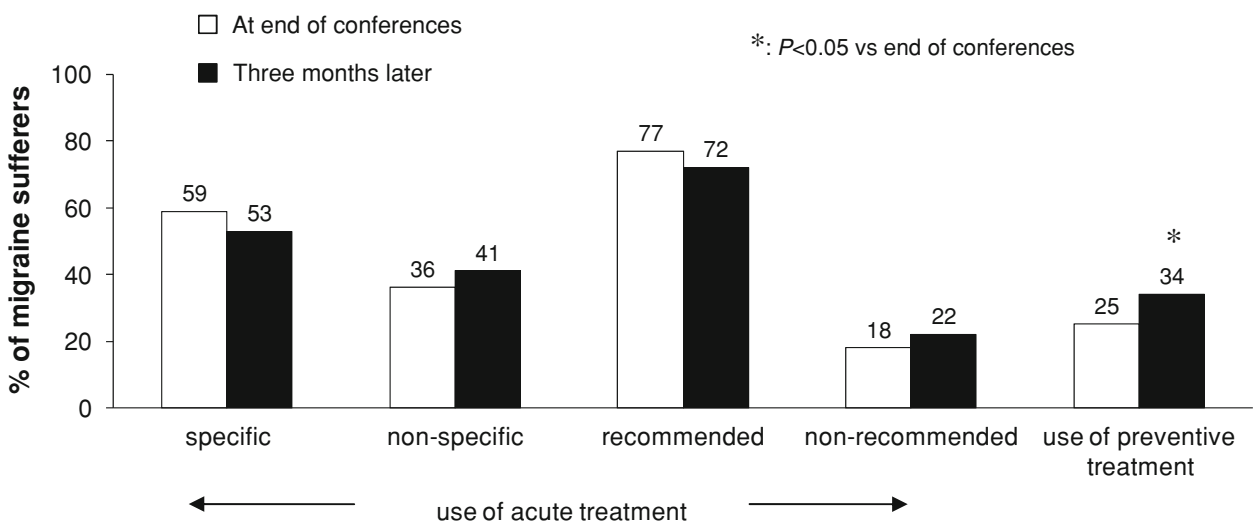


were recruited by a mailing to more than 8,000 households listed in the registries of a group of ten general practitioners and posters in the physicians' waiting rooms. Migraine sufferers were first identified by the ID Migraine screening test [17], then migraine was diagnosed according to the ICHD-II criteria during a consultation. The authors expected to recruit 1,300-1,500 migraine sufferers via the personal mails addressed: in reality, only 195 headache sufferers consulted as a result of the sensitization campaign. Most participants (92\%) were migraine sufferers and $73 \%$ were diagnosed for the first time. As in Tour de France of migraine, the migraine sufferers who actually consulted were those with severe headaches: mean HIT-6 score was $61.5 \pm 7.6$ and subjects suffered a mean number of $8.4 \pm 9.0$ days of headache per month with a mean pain intensity of $8.6 \pm 1.5$ on a scale of $0-10$. Like ours, this sensitization campaign failed to bring a wide range of undiagnosed headache sufferers into medical care: patients with high-grade disability suffer migraine attacks of severe intensity and use multiple drug units to treat the attacks [14]. As underlined by the authors of the Casilino study, more widespread use of rapid identification of potential migraine sufferers by the ID Migraine test might be very useful for a large recruitment of migraine patients to medical care. Interestingly, $92 \%$ of patients identified using ID Migraine in the Casilino study were true migraine sufferers, versus $96 \%$ of headache sufferers attending the conferences in the Tour de France of migraine, indicating that a wide public information campaign mostly reaches migraine sufferers and less subjects with other categories of common headache disorders such as tension-type headaches.

As expected, migraine sufferers not medically followed up used fewer specific and recommended treatments of migraine, more frequently took large number of medication units for an attack, and fewer received a preventive treatment of migraine. The conferences did not seem to have influenced the therapeutic behaviour of the migraine sufferers. This is in keeping with data from a pharmaceutical campaign conducted in Denmark targeting the inappropriate use of triptans which showed that intensive information of pharmacy staff had no significant impact after 6 or 9 months on the patients' consumption of triptans [18].

Nonetheless, 3 months after the conferences, more migraine sufferers than initially received a preventive treatment of migraine ( $34 \mathrm{vs.} 25 \%$ ), as a possible result of the $27 \%$ of initially migraine sufferers without medical follow-up who consulted after the conferences or of specific request from consulting migraine sufferers after the sensitization campaign. Concomitantly, mean migraine impact on daily life as assessed by the HIT-6 score decreased slightly but significantly $(-3.5 ; P<0.05)$ during the period in the whole group of migraine sufferers.
There was no significant difference in impact change following the sensitization campaign whether migraine sufferers had consulted or not, however, which makes difficult the identification of the cause of migraine impact alleviation.

The Tour de France of migraine sensitization campaign has some limitations, however. It concerned mainly the urban population of France, as it was conducted in only six large towns in France. However, the prevalence of migraine does not appear to differ according to community size [13]. The proportion of responding subjects was quite low and probably not totally representative of the whole population of migraine sufferers since most participants were severe migraine sufferers and already enrolled in the healthcare system. On the other hand, a strength of the survey is the relatively high proportion of migraine sufferers $(85 \%)$ that could be contacted again 3 months after the sensitization campaign, with no apparent bias due to population attrition. To extend this type of migraine sensitization campaign, it may be interesting to develop other ways of communication as the Internet, information close to the pharmacists, etc.

In conclusion, the Tour de France of migraine campaign that intended to sensitize headache sufferers to consult mainly reached severe migraine sufferers who were already medically followed up. Nevertheless, the sensitization campaign had some positive influence on migraine management, in that some migraine sufferers previously without medical follow-up actually consulted during the 3 months following the sensitization programme, more of them received a preventive treatment, and a slight but significant decrease in migraine impact on daily living was observed. No relationship between these changes could be firmly established, however. Other strategies should thus be developed to reach a more general population of migraine sufferers. Public information and individual patient's therapeutic education are indeed becoming an essential part of the treatment of chronic diseases. It is now largely recommended that the combination of both, complementary but not equivalent, should integrate the therapeutic strategy of chronic diseases, such as asthma, diabetes and migraine $[6,19,20]$. This strategy would be particularly important for migraine, a condition where patient defeatism and unawareness of the current effective treatments of migraine might be at least in part responsible for the high rate of exclusion from medical care.

Acknowledgments The authors would like to express their appreciation to Pascaline Lavalade and to acknowledge Dr. Michèle Barège, Dr. Frédéric Brudon, Dr. Dominique Deplanque, Dr. Xavier Douay, Dr. Nelly Fabre, Dr. Ghislain Malanda, Dr. Marie-Anne Mackowiack-Cordoliani, Dr. Hélène Massiou, Prof. André Pradalier, Dr. Anne Revol, Dr. Jean-Denis Turc and Dr. Louis Vernier for their outstanding part in conducting the conferences. The campaign was 
subsidized by a grant from the national 2006 Migraine Expert Group funded by AstraZeneca, and by a grant from Janssen-Cilag.

Conflict of interest This study was funded by a grant from Astra Zeneca. Competing interests: none.

\section{References}

1. Stovner LJ, Hagen K, Jensen R, Katsarava Z, Lipton R, Scher A, Steiner T, Zwart JA (2007) The global burden of headache: a documentation of headache prevalence and disability worldwide. Cephalalgia 27:193-210. doi:10.1111/j.1468-2982.2007.01288.x

2. Lucas C, Géraud G, Valade D, Chautard MH, Lantéri-Minet M (2006) Recognition and therapeutic management of migraine in 2004, in France: results of FRAMIG 3, a French nationwide population-based survey. Headache 46:715-725. doi:10.1111/ j.1526-4610.2006.00430.x

3. Lipton RB, Scher AI, Kolodner K, Liberman J, Steiner TJ, Stewart WF (2002) Migraine in the United States: epidemiology and patterns of health care use. Neurology 58:885-894

4. Lantéri-Minet M, Massiou H, Nachit-Ouinekh F, Lucas C, Pradalier A, Radat F, Mercier F, El Hasnaoui A (2007) The GRIM2005 study of migraine consultation in France. I. Determinants of consultation for migraine headache in France. Cephalalgia 27:1386-1397. doi:10.1111/j.1468-2982.2007.01426.x

5. Diener HC, Limmroth V (2004) Medication-overuse headache: a worldwide problem. Lancet Neurol 3:475-483. doi:10.1016/ S1474-4422(04)00824-5

6. World Health Organization (2007) Lifting the burden: the global campaign to reduce the burden of headache worldwide. J Headache Pain 8:S1

7. Harpole LH, Samsa GP, Jurgelski AE, Shipley JL, Bernstein A, Matchar DB (2003) Headache management program improves outcome for chronic headache. Headache 43:715-724. doi: 10.1046/j.1526-4610.2003.03128.x

8. Rothrock JF, Parada VA, Sims C, Key K, Walters NS, Zweifler RM (2006) The impact of intensive patient education on clinical outcome in a clinic-based migraine population. Headache 46:726-731. doi:10.1111/j.1526-4610.2006.00428.x

9. Headache Classification Subcommittee of the International Headache Society (2004) The international classification of headache disorders, 2nd edition. Cephalalgia 24(Suppl 1):9-160. doi: 10.1111/j.1468-2982.2003.00824.x

10. Kosinski M, Bayliss MS, Bjorner JB, Ware JE Jr, Gardber WH, Batenhorst A, Cady R, Dahlöf CG, Dowson A, Tepper S (2003) A six-item short-form survey for measuring headache impact: the HIT-6. Qual Life Res 12:963-974. doi:10.1023/A:1026119331193

11. Haute Autorité de Santé (2002) Professional recommendation. Diagnostic and therapeutic management of migraine in adults and children: clinical and economical aspects. Available at http://www.has-sante.fr/portail/display.jsp?id=c_272212 (in French)

12. Géraud G, Lantéri-Minet M, Lucas C, Valade D, French Society for the Study of Migraine Headache (SFEMC) (2004) French guidelines for the diagnosis and management of migraine in adults and children. Clin Ther 26:1305-1318. doi:10.1016/ S0149-2918(04)80161-9

13. Lantéri-Minet M, Valade D, Géraud G, Chautard MH, Lucas C (2005) Migraine and probable migraine-results of FRAMIG 3, a French nationwide survey carried out according to the 2004 IHS classification. Cephalalgia 25:1146-1158

14. Lucas C, Chaffaut C, Artaz MA, Lanteri-Minet M (2005) FRAMIG 2000: medical and therapeutic management of migraine in France. Cephalalgia 25:267-279. doi:10.1111/j. 1468-2982.2004.00851.x

15. Baszanger I (1998) Sociological aspects. Managing migraine: experience, knowledge and patient information. In: Expertise collective INSERM. La migraine. Les Editions INSERM, Paris, pp 251-263 (in French)

16. Di Piero V, Altieri M, Conserva G, Petolicchio B, Di Clemente L, Hettiarachchi J, "General Practitioners' Co-operative" of the Casilino district of Rome (2007) The effects of a sensitisation campaign on unrecognised migraine: the Casilino study. J Headache Pain 8:205-208. doi:10.1007/s10194-007-0395-6

17. Lipton RB, Dodick D, Sadovsky R, Kolodner K, Endicott J, Hettiarachchi J, Harrison W, ID Migraine validation study (2003) A self-administered screener for migraine in primary care. Neurology 61:375-382

18. Søndergaard J, Foged A, Kragstrup J, Gaist D, Gram LF, Sindrup SH, Muckadell HU, Larsen BO, Herborg H, Andersen M (2006) Intensive community pharmacy intervention had little impact on triptan consumption: a randomized controlled trial. Scand J Prim Health Care 24:16-21. doi:10.1080/02813430500444916

19. Ruoff G, Urban G (2004) Treatment of primary headache: patient education. In: Standards of care for headache diagnosis and treatment. National Headache Foundation, Chicago, pp 22-26

20. Haute Autorité de Santé (2007) Professional recommendation. Therapeutic patient education. Definition, purposes and organization. Available at http://www.has-sante.fr/portail/upload/docs/ application/pdf/etp_definition_finalites_recommandations_juin_ 2007.pdf (in French) 